\title{
Aspects regarding numerical models for safe evacuation of people, in the current pandemic context
}

\author{
Marius Cornel Şuvar ${ }^{1 *}$, Vlad Mihai Păsculescu ${ }^{1}$, Alin Irimia $^{1}$ and Dragoș Păsculescu ${ }^{2}$ \\ ${ }^{1}$ National Institute for Research and Development in Mine Safety and Protection to Explosion - \\ Insemex Petroșani, 32-34 Gen. Vasile Milea Str., Petroșani, Romania \\ ${ }^{2}$ University of Petroșani, Department of Mechanical, Industrial and Transport Engineering, 20 \\ University Street, Petroșani, Romania
}

\begin{abstract}
In everyday life, several situations can be mentioned in which a building or a complex of buildings may require emergency evacuation: fires, chemical leaks, release of toxic or explosive gases, explosions, violent behavior, or threats with weapons/bombs.

To calculate the time needed for building evacuation, numerical models are used to simulate this process of movement of groups of people, in a closed physical space. Algorithms for access path and exit selection use both properties of the crowds model and the individual interaction between event and people.

The pandemic context has raised several questions about the safe use of buildings, given the presence of the risk of disease transmission. The policies adopted in the last year regarding the use of buildings, establishing access flows, and social distance, vary within great limits, being specific to each state and based on the analysis of the virus transmission rate rather than on risk assessments at the building level.

The paper aims to present the main challenges to which the models of emergency evacuation, must respond, especially those considering social distancing and interaction between individuals, within a given distance, all to minimize the risk of disease transmission during the evacuation process of the building.
\end{abstract}

\section{Introduction}

In the last years, research on the evacuation of people in emergencies has seen an unprecedented development, fire safety specialists adopting new methods of studying this issue, based mainly on mathematical analysis and computer simulations. Numerical analysis methods are based on the mathematical model, on which the current parameters of the evacuation process are converted into mathematical equations, which are to be solved using the computational technique. These models are classified into macroscopic models, respectively microscopic models [1, 2]. Microscopic models (cellular automaton model, multi-lattice model, and probabilistic models) take into account the individual and behavioral

${ }^{*}$ Corresponding author: marius.suvar@insemex.ro 
characteristics of evacuees, often referred to as agents, as well as the interactions between them during the evacuation process. For these reasons, the choice of optimal escape routes cannot be easily achieved, especially in the case of large buildings. Macroscopic models ignore the behavior of individuals involved in the evacuation process, being based on flow network models, which can more quickly solve the problem of choosing the optimal escape routes in a state of emergency [3].

Computational evacuation models offer diverse sets of techniques and methods covering a wide range of applicability, from relatively homogeneous flows of occupants to autonomous agents, which move freely in a three-dimensional space. Currently, more than 30 computer models are known focused on studying the evacuation process from buildings, many of which can simulate evacuation even from other types of structures. Simultaneously with the development of these models, there are in the literature numerous new researches that propose or study evolved numerical models, optimized for evacuation processes from a wide range of applications. Such models are capable of dealing not only with evacuation in the event of a fire but also in the event of any imminent threat or danger to which a group of the population may be exposed: evacuation in non-emergency situations, evacuation from buildings, following fire or storms, macro-scale evacuation in case of natural disasters or industrial incidents, traffic accidents, etc.

In the field of building safety engineering, to calculate the time needed to evacuate buildings in case of emergency, numerical models are used that simulate this process of movement of groups of people, in a closed, delimited physical space, while tracking the trajectory and the position of each individual, under the influence of specific forces, characteristics and strategies for evacuation. Algorithms for access path and exit selection use both properties of the crowds model and the individual interaction between the event and the people.

Currently, concern about the continued spread of the Covid-19 pandemic, and the challenge of maintaining a low rate of spread of this virus have led to the adoption of a series of measures of unexpected magnitude, to ensure the safety and health of people. The pandemic context has raised several questions about the safe use of buildings, given the presence of the risk of disease transmission among their occupants. The policies adopted in the last year regarding the use of buildings, establishing access flows, and social distance, vary within great limits, being specific to each state and based on the analysis of the virus transmission rate rather than on risk assessments at the building level.

After the initial lockdown period, in the acute phase of the pandemic, in which the use of a large number of buildings and public spaces was almost completely reduced, the gradual resumption of activities is a new challenge in terms of use and user experiences. In this context, the traditional design of buildings, their classic interior compartmentation, well known and applied over time, gives way to new conditions, sometimes incompletely tested, which must offer the possibility of normal course of activities, while ensuring a risk of disease and transmission of the virus as little as possible.

A recent report [4] presents an unprecedented situation in the case of office buildings and activities, with an increase in the number of people adopting work from home and companies, who are required to redesign workspaces and access circuits in order to comply with social, physical distancing, to avoid human congestion and to ensure a high level of air quality and good recirculation. These aspects are just one side of the new framework of measures that will require strong changes in the functionality of companies, the development of work processes, the definition of productivity, the development of teamwork, etc.

The process of rethinking and redesigning public spaces and future uses modality faces two major challenges: on the one hand, the lack of historical data to support the new design decisions and to allow the evaluation of projects also in terms of risk exposure to viral infection. Although new regulations and protocols have emerged in this direction, there is 
still a high degree of uncertainty about the extent to which general assumptions about social distancing are taken into account and respected. On the other hand, it is necessary for this process of rethinking the future to take place in a short term, in the order of months, to reensure again the productivity of activities, the profitability of buildings, and the access to public spaces and cultural institutions.

The paper aims to present the main challenges to which the models of emergency evacuation, must respond, especially those considering social distancing and interaction between individuals, within a given distance, all to minimize the risk of disease transmission during the evacuation process of the building.

\section{Computerized models for emergency evacuation - theoretical aspects}

Simulation of human behaviour in emergencies (e.g., in fire) is one of the biggest challenges in designing buildings, complex structures or industrial sites (nuclear power plants, chemical plants, hazardous waste landfills, etc.), in an attempt to obtain a high security level for the occupants' lives. Fire safety engineers have reached a consensus when they say that the results of computer simulations (of fire and/or occupant evacuation) are valuable input in assessing the fire safety performance of buildings, using analytical methods of risk.

The differences imposed by the behavioral aspects of the evacuated occupants can be probabilistically simulated in the evacuation models, through the distribution functions. An important component of assessing the safety of buildings in emergencies refers to the calculation of the time required for occupants to reach a safe place, respectively the time required to leave the space threatened by the event. In the case of fire safety, this time is called the required safe egress time - RSET, and the available safe egress time-ASET, where RSET $<$ ASET to permit the occupant to reach a safe place, without adverse effects. For modeling the ignition and development of fire and smoke migration, a variety of numerical models are used (Gaussian models, CFD - Computational Fluid Dynamics models, etc.) [5, $6]$.

For example, in the computational model for fire and evacuation FDS+EVAC, the required safe egress time (RSET) may be expressed as:

$$
R S E T=t_{\text {pre }}+t_{\text {depl }}=\left(t_{\text {det }}+t_{\text {reac }}\right)+t_{\text {depl }}
$$

where: $t_{\text {pre }}$ - pre-evacuation time, beginning from the moment of fire ignition and ending when the occupants start moving to the nearest exist, being variable and dependent by the occupants' behaviour and the functional characteristics of the building. This time is composed by:

- the detection time, $t_{d e t}$ (from the fire outbreak to the moment of detection): the occupants continue their initial activities (study, work on the computer, etc.), expressed in seconds (s);

- the reaction time, $t_{\text {reac }}$ (during which people make decisions about evacuation): occupants perform a wide range of activities, investigate the source of fire, turn off computers, collect personal belongings, group with close people, look for escape routes, alert other people try to extinguish the fire, or may be passive - expressed in seconds (s);

- $\quad$ travel time, $t_{\text {depl }}-$ time in which the people move to a safe zone, expressed in seconds (s).

Various numerical models can be used for evacuation in emergencies, from simple models, in form of equation (e.g. the hydraulic model proposed by SFPE, which can be solved using a spreadsheet), to models based on complex algorithms, integrated into computer software application (continuous models, discrete models, hybrid models, etc.). Initially, the 
vast majority of these numerical models were deterministic, but in the recent decades, there has been an increase in the use of probabilistic models as part of the security system design process, under the influence of uncertainty factors, especially for complex buildings and industrial areas.

One of the main issues associated with evacuation models is that they may represent certain aspects of human behavior based on theoretical assumptions rather than behavioral data [7]. In fact, while evacuation models have largely improved their capabilities over the years in representing the physical aspects associated with pedestrian flows, to date, the representation of behavioral aspects of an evacuation highly relies on the model user expertise rather than the methods adopted. For this reason, there is an agreement in the evacuation modeling community that model validation should be one of the primary areas of evacuation research in the future [7 - 10].

We can say that the response to the action of fire or other disasters is the result of undertaking a process. People in a building or groups of occupants are involved in a decisionmaking process (a series of steps, or activities) before responding to the threat, based on information gathered from the environment. Before any rescue action is decided, subjects must gather information, clues, or evidence (visual, auditory, or olfactory perception), pay attention to it, and understand it correctly. It is therefore very important to specify that, through design or evacuation plans, it must be ensured that these three processes are carried out.

Actions are taken by the occupants as a consequence of the decision-making process. In situation when the occupants of the building do not perceive the risk or the possible threat, it is very likely that they will not pay attention to the clues and they will continue their activities normally, delaying the decision-making process and initiating rescue action. Similarly, in situations where they perceive the threat as uncertain or unclear, occupants may engage in activities to obtain more information, which also delays rescue decisions. Research has shown that providing alerting/alarming information in various ways (visual, auditory) removes the need to search for additional information and clues, facilitating the decisionmaking process and shortening the time required to travel to a safe zone.

In principle, the implemented mathematical model treats each person who evacuates (called agent in the program) as a single entity, whose time-dependent displacement is characterized by an equation. Each agent has its own properties and evacuation strategies. Agents are subjected to forces and moments of contact that are the consequence of displacement and psychological behavior.

The equations of motion, due to the granted translation and rotation degrees of freedom, are solved with methods specific to the dynamics of dissipative particles; thus, the model uses continuous time and space to trace the trajectories of agents. Agents face forces and moments of contact, their own psychology, forces and motivational moments.

For example, the algorithm for the agent movement, in FDS + EVAC, one of the most well-known and used computer models, is based on Helbing's method, being implemented in the FDS program environment by Korhonen, Hostikka and Keski-Rahkonen and subsequently modified by Langston, which replaces the representation of the human individual with a single circle, with one through three circles (fig. 1) approximating the elliptical section of the cross-section of the human body. FDS + EVAC allows the modeling of situations characterized by large masses of occupants, as well as the interaction between simulation of evacuation and fire development [7]. 


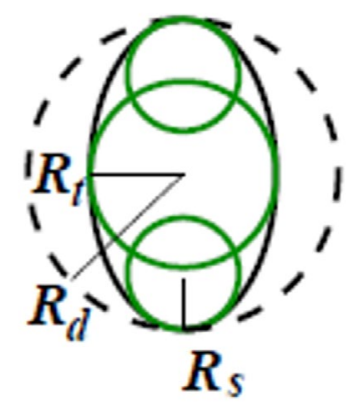

Fig. 1. Representation of human body (basic representation) in FDS+EVAC model

\subsection{Agent movement model}

The agent movement algorithm in occupant evacuation models has as a starting point, the method developed by Helbing's group, introducing the so-called social force, to keep reasonable distances to walls and other agents. The model uses the laws of mechanics to follow the trajectories of the agents during the calculation. Each agent follows its own equation of motion:

$$
m_{i} \frac{d^{2} x_{i}(t)}{d t^{2}}=f_{i}(t)+\xi_{i}(t)
$$

where: $x i(t)$ - the position of agent $i$ at time $t, f_{i}(t)$ - the force exerted on agent $i$ by the surroundings, $m_{i}$ - the agent's mass, constant during the simulation, and $\xi_{i}(t)$, small random fluctuation force. The velocity of agent is given by: $v_{i}(t)=d x_{i} / d t$.

The force exerted on the agent $i$ has many components:

$$
f_{i}=\frac{m_{i}}{\tau_{i}}\left(v_{i}^{0}-v_{i}\right)+\sum_{j \neq i}\left(f_{i j}^{s o c}+f_{i j}^{c}+f_{i j}^{a t t}\right)+\sum_{w}\left(f_{i w}^{s o c}+f_{i w}^{c}\right)+\sum_{k} f_{i k}^{a t t}
$$

Where: the first sum describes agent-agent interactions, the second sum, agent-wall interactions, and the terms in the last sum is used to describe agent-environment interaction, like the fire-agent repulsion. Figure 2 shows the concept of social force, as applied in FDS+EVAC model [11]:

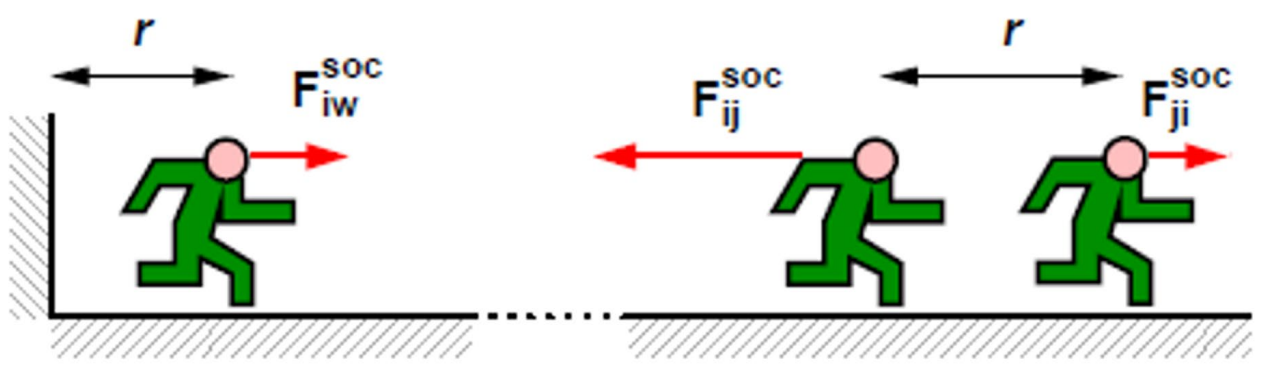

Fig. 3. The concept of the social force. 
When applying the algorithm for calculating the safe evacuation time and choosing the optimal paths, the numerical model takes into account a multiple series of forces, among which can be listed: the social force defined by the interactions between agents, the social force for the interaction between the agent and walls, the force of physical contact between agents and between agents and walls, radial elastic force, friction force, forces of attraction/repulsion between agents, to simulate group, adult-child or adult-helpless person behavior $[12,13]$, etc.

The effects of smoke and toxicity on agent travel speeds are implemented by the movement model underlying evacuation algorithms. Thus, the exit selection algorithm uses smoke density in calculating exit door visibility and in classifying doors according to preference. Agents are assimilated to the flow of an incompressible, two-dimensional fluid, determined by the boundary conditions imposed by the geometry of the building and the chosen exit door (which behaves like a fan that pulls the fluid out of the occupied field at a given time). This solution produces a field pointing to the specified output, which will always guide agents to that output. The route chosen for the movement of agents will not be the shortest, but, in most cases, it will be close to it. The field will guide more agents to the wider escape routes than to the narrower ones because this field is a specific solution for incompressible flow.

\section{Aspects regarding modeling assumptions on occupant exposure}

Since the mode of transmission of severe acute respiratory syndrome coronavirus 2 (SARS$\mathrm{CoV}-2$ ) is not fully known to date, one of the accepted assumptions is that of similarity to severe acute respiratory syndrome coronavirus 1 (SARS-CoV-1), in which the spread was proved to be achieved by: physical contact, fluid droplets and by airborne routes [16]. Due to this uncertainty dictated by the mechanism of transmission of the virus in the Covid-19 pandemic, E. Ronchi and the team [15] propose 5 assumptions on occupants exposures in a building, which evacuation models should take into account:

- Exposure by physical contact;

- Exposure considering social distance radius;

- Exposure by face-to-face contact within a social distance radius;

- $\quad$ Exposure by being in the same room;

- Exposure by being in the same building.

\subsection{Exposure by physical contact}

We can talk about occupant exposure by physical contact when the people share the same closed space and are in direct physical contact to each other (agents touch each other). It can be stated that this exposure is characteristic of most numerical models based on the crowd movement (groups of people), which use the representation of individual agents through circles or ellipses, so that their spatial coordinates overlap within a certain radius, corresponding to their dimensions. This assumption requires from a crowd model the information regarding the agents' trajectories over time and their dimensions.

\subsection{Exposure considering social distance radius}

This hypothesis of virus transmission involves the existence of a number of persons located at a physically user-defined distance ( 1 to $2 \mathrm{~m}$ ). The center of the social distance radius can 
be assumed to be the center of the modeled occupant or another point on its outer border. This can be implemented by checking the agent's spatial coordinates and evaluating its positioning within the specified interaction range. This can conservatively consider that if one agent has at least one part of the body within the social distance radius, it is counted. This hypothesis requires from the model of information about occupant trajectories over time.

\subsection{Exposure by face-to-face contact within a social distance radius}

The hypothesis presumes the face-to-face contact within the social distance radius, within a given angle of interaction, defined by the user. Ronchi et Lovreglio propose the use of the polar coordinates $\propto_{i j}(t)$ and $\rho_{i j}(t)$ to define the position of agent $j$ in polar space, relative to agent $i$, where $\rho_{i j}$ changes over time. It can take values from 0 (agents touch each other), up to $\rho_{i j}=R_{i}$, at the limit of the social distance radius, so $\rho_{i j}=\left[0, R_{i}\right] \cdot \propto_{i j}(t) \cdot \beta_{i j}(t)$ represents the orientation of agent $j$ in the polar space defined by the agent $i$, taking values from zero (agent $j$ is facing the agent $i$ ) and $\pm \pi$ (agent $j$ is right behind agent $i$ ). $\beta_{i j}(t)$ parameter can be used to evaluate how many agents are face-to-face contact within a social distance radius, at a given time. This assumption requires from the crowd model the information concerning both the occupant trajectories over time, as well as the direction of movement of each agent, to know the face orientation.

\subsection{Exposure by being in the same room}

This hypothesis involves the exposure of occupants sharing the same room or compartment. In order to be considered, this assumption requires from the model information about the number of occupants in each room, at each time steps.

\subsection{Exposure by being in the same building}

Exposure of occupants in an enclosed space, in this case, assumes that agents are locating in the same building and is the simplest change in terms of model implementation, requiring only existing information, related to the number of occupants in the building and its geometry (interior compartmentation mode).

The 5 modes of occupant exposure that should be considered and implemented in computer models for people evacuation simulation in emergencies are presented in fig. 2.

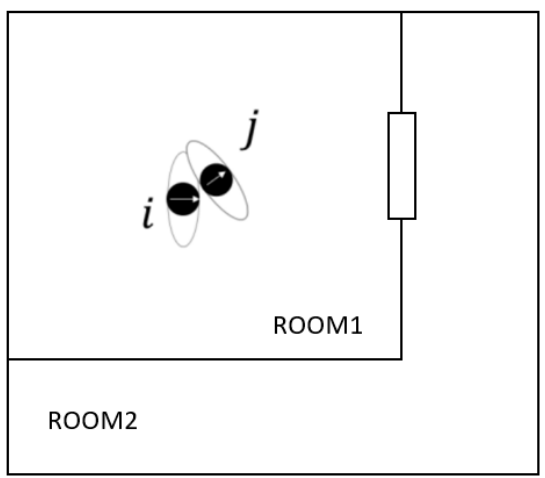

a) Exposure by physical contact

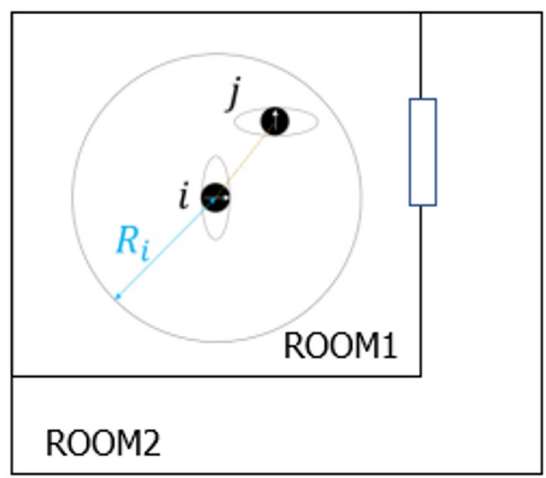

b) Exposure considering social distance radius 


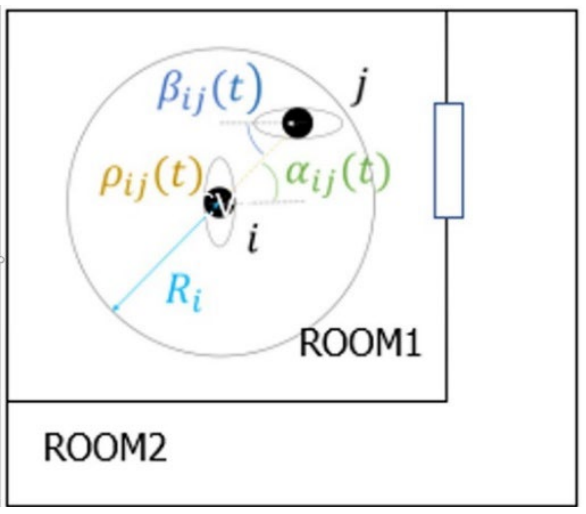

c) Exposure by face-to-face contact within a social radius

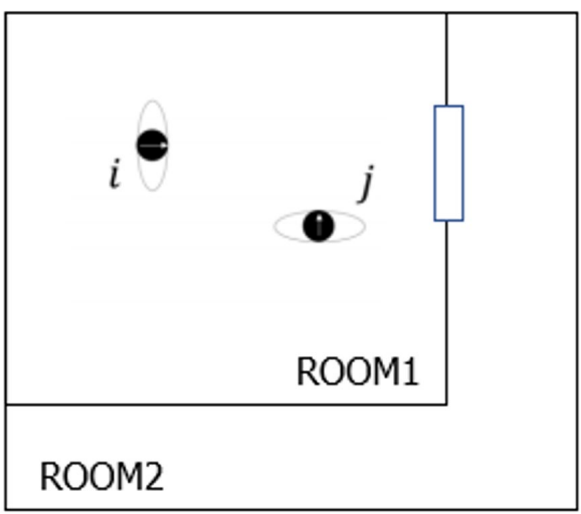

d) Exposure by being in the same room

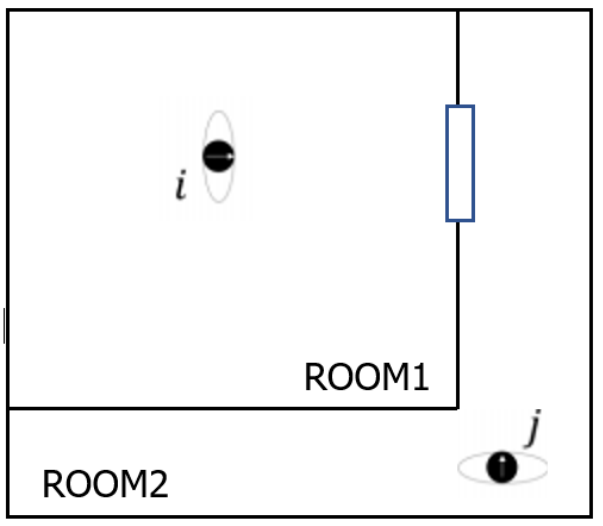

e) Exposure by being in the same building

Fig. 2. Assumptions on occupant exposure considering two different rooms and a door between them

\section{Conclusions}

Based on the information above, the following conclusion can be drawn:

- Current evacuation management plans and future building design in terms of fire and emergency evacuation will need to be reviewed to incorporate measures to limit the occupant exposure to COVID-19 or other pandemic situations.

- No current evacuation models take into account this new threat; probably, in the next years, their algorithms will be enhanced to take into consideration the physical distancing and occupant exposure, noting preservation of life will always be the first priority.

- The crowd models must be redesigned to support risk assessment based on occupant exposure in confined spaces during pandemics. These new approaches allow more informed decisions concerning building access restrictions during pandemics by performing a quantitative assessment of occupant exposure. 


\section{Acknowledgements}

This paper was developed within the Nucleu-Programme, carried out with the support of the Ministry for Research, Innovation and Digitalization, project no. PN-19-21-01.02, Development of institutional capability for technical expertise of fire type events for residential and industrial environments, by computerized simulation (in Romanian).

\section{References}

1. H. Hamacher, S. Tjandra, Mathematical modelling of evacuation problems: A state of the art (Pedestrian and Evacuation Dynamics, Springer, Berlin, 2002)

2. C. Liu, Z-1. Mao and Z-M. Fu, Procedia Engineering 135, 12-18 (2016)

3. E. Vilar, F. Rebelo, and P. Noriega, Applied Ergonomics 44, 618 (2013)

4. McKinsey Report Reimaging the office and work life after COVID-19. Available on https://www.mckinsey.com/business-functions/organization/our-insights/reimagining-the-officeand-work-life-after-COVID-19

5. D.R. Blackmore, M.N. Herman, J.L. Woodward, J. Hazard. Mater. 6, 107-128 (1982)

6. M. Siddiqui, S. Jayanti, T. Swaminathan, J. Hazard. Mater. 209-210, 177-185, (2012)

7. S.M.V. Gwynne, L.M. Hulse, M.J. Kinsey, Fire Technology 52, 775-800 (2015)

8. Y. Cheng, X. Zheng, Communications in Nonlinear Science and Numerical Simulation 66, 216225 (2019)

9. E. Ronchi, M. Kinsey, Evacuation Models of the Future: Insights from an Online Survey on User's Experiences and Needs, Advanced Research Workshop Evacuation and Human Behaviour in Emergency Situations, Santander, Spain, 145-155 (2011)

10. M.C. Suvar, I. Kovacs, V.M. Păsculescu, N.I. Vlasin, G.D. Florea, Environmental Engineering and Management Journal 18 (4), 921-928 (2019)

11. T. Korhonen, Fire Dynamics Simulator with Evacuation: FDS+Evac. Technical Reference and User's Guide (VTT, Finland, 2018). Available on http://virtual.vtt.fi/virtual/proj6/fdsevac/documents/FDS+EVAC_Guide.pdf

12. E.D. Kuligowski, Human Behavior in Fire, SFPE Handbook of Fire Protection Engineering (Springer, New York, 2016)

13. Y. Zheng, B. Jia, X. Li, R. Jiang, Safety Science 92, 180-189 (2017)

14. R. Lovreglio, M. Spearpoint, and M. Girault, Reliability \& Engineering System Safety, 185, 1$566(2019)$

15. E. Ronchi, R. Lovreglio, Safety Science 130, 104834 (2020)

16. I.T. Yu, Y. Li, T.W. Wong, W. Tam, A.T. Chan, J. Lee, D.Y. Leung, and T. Ho, New England Journal of Medicine 350 (17), 1731-1739 (2004) 